\title{
IMPACTOS AMBIENTAIS DA PRODUÇÃO AGRÍCOLA DO TABACO ORGÂNICO E CONVENCIONAL NO SUL DO BRASIL
}

\section{ENVIRONMENTAL IMPACTS OF ORGANIC AND CONVENTIONAL TOBACCO AGRICULTURAL PRODUCTION IN SOUTH BRAZIL}

\author{
Priscila Fernandes de Oliveira*E-mail: prifernandes2010@hotmail.com \\ Ana Letícia Zappe* E-mail: anazappe@gmail.com \\ Ricardo Boethcher ${ }^{*}$ E-mail: boettcher.ricardo@yahoo.com \\ Diosnel Antonio Rodriguez Lopez*E-mail: dlopez@unisc.br \\ "Universidade de Santa Cruz do Sul (UNISC), Santa Cruz do Sul, RS.
}

\begin{abstract}
Resumo: Foi realizado uma Análise de Ciclo de Vida da produção de tabaco orgânico e convencional no sul do Brasil, com o objetivo de avaliar os impactos ambientais associados às etapas de produção de mudas, lavoura, cura e transporte. Os dados utilizados para a caracterização foram obtidos através da aplicação de questionários aos produtores e transportadores e também empregados dados de produção de 12 mil produtores de tabaco, disponibilizados por uma empresa beneficiadora da região. A avaliação dos impactos foi realizada por meio da Análise de Ciclo de Vida. A unidade de referência foi a produção de uma tonelada de tabaco seco. Foram analisadas seis categorias de impacto, sendo Potencial de Aquecimento Global, Acidificação Terrestre, Ecotoxicidade Terrestre Eutrofização da água doce, Uso da terra e Escassez de recursos fósseis. Os resultados conseguiram transparecer as etapas de maior impacto nas duas formas de produção. Na comparação de resultados entre os sistemas de plantio em cada categoria de impacto foi possível verificar que o plantio orgânico apresenta impactos maiores para as categorias de acidificação terrestre, potencial de aquecimento global e uso da terra. Por sua vez, o plantio convencional teve maiores valores totais de impactos para as categorias de potencial de eutrofização da água doce e escassez de recursos fósseis.
\end{abstract}

Palavras-chave: Ciclo de vida. Impactos ambientais. orgânico. tabaco. produção.

Abstract: A Life Cycle Analysis of organic and conventional tobacco production in southern Brazil was carried out to evaluate the environmental impacts associated with the stages of seedling production, tillage, curing and transportation. The data used for the characterization were obtained by applying questionnaires to producers and transporters and also used production data from 12,000 tobacco producers, provided by a beneficiary company in the region. Impact assessment was performed through Life Cycle Analysis. The reference unit was the production of one ton of dry tobacco. Six impact categories were analyzed: Global Warming Potential, Terrestrial Acidification, Terrestrial Ecotoxicity, Freshwater Eutrophication, Land Use and Fossil Resource Shortages. The results were able to show the stages of greatest impact on both forms of production. Comparing the results between the planting systems in each impact category it was possible to verify that the organic planting presents larger impacts for the terrestrial acidification, global warming potential and land use categories. In turn, conventional planting had higher total impact values for the categories of freshwater eutrophication potential and scarcity of fossil resources.

Keywords: Life cycle. Environmental impacts. Organic. Tobacco. Production. 


\section{INTRODUÇÃO}

A introdução A planta Nicotiana tabacum, nome científico do tabaco ou fumo, passou a ser conhecida e utilizada no mundo inteiro. A disseminação da produção se fez através dos marinheiros e soldados, que utilizavam o mesmo durante os longos períodos das viagens e através das expedições portuguesas e espanholas, que levaram a planta para Europa, difundindo-a nos outros países europeus, na África e no Oriente (NARDI; NARDI, 1996).

Sua origem é cogitada nos Vales dos Andes Bolivianos, alastrando-se através das migrações indígenas para o território brasileiro. O cultivo da planta no sul do Brasil foi realizado devido os imigrantes alemães, os quais tiveram um papel fundamental na inserção do cultivo do tabaco na região. A difusão da cultura é atribuída à grande disponibilidade de mão-de-obra na época, destacando a cidade de Santa Cruz do Sul/RS como centro da produção de tabaco na região (DUTRA; HILSINGER, 2013).

O plantio de tabaco é caracterizado como uma commodity agrícola e hoje proporciona uma fonte de renda única a diversos produtores, contribuindo para o desenvolvimento dos municípios. Entre os anos de 1977 a 2004, a produção de tabaco no Brasil cresceu $260 \%$, devido à uma demanda crescente e para suprir as necessidades de consumo da sociedade atual (TILMAN et al., 2001; Garnett, 2011).

Os custos ambientais, atrelados a cadeia produtiva de commodities, podem ser caracterizados pela suas contribuições sobre os impactos ambientais, como esgotamento dos recursos naturais, emissões de gases do efeito estufa, perda da fertilidade do solo, escassez da água e a liberação de grandes quantidades de nutrientes e outros poluentes que afetam os ecossistemas (MCMICHAEL et al., 2007). Desta forma, o cenário atual da produção de tabaco inclui os problemas mais conhecidos associados ao sistema de produção, os quais incluem danos ao meio ambiente e riscos à saúde (VARGAS; OLIVEIRA, 2012).

Por outro lado, auferir os encargos sociais, econômicos e ambientais de um sistema produtivo é uma crescente demanda, a fim de fornecer maior ênfase em estudos aplicados, devido à importância da construção de sistemas produtivos dentro das premissas da sustentabilidade. Para (FAO, 2015), se nada mudar na forma como produzimos e consumimos, os impactos ambientais se tornarão cada vez mais severos. 
O desafio se consolida na aplicação da abordagem de Análise de Ciclo de Vida (ACV) para a avaliação das etapas de produção agrícola do tabaco, tanto orgânico quanto convencional, levando em consideração, sobretudo, sua complexidade e a agregação de variáveis ambientais nessa análise. Com o intuito de contribuir podendo relacionar conceitos teóricos à prática, permitindo assinalar pontos críticos da produção e assim ajudar na aplicação de melhorias em questões específicas.

Esta ferramenta compila um inventário das trocas ambientais relevantes ao longo de ciclo de vida do processo e avalia os impactos ambientais potenciais associados com essas trocas (WIEDMANN et al., 2013). Para (BRENTRUP et al., 2004) ACV é uma abordagem apropriada para a investigação de impactos ambientais em produtos. A aplicação da ferramenta da Avaliação de Ciclo de Vida (ACV) à agricultura é relativamente recente e complexa, mas se destaca pela abrangência e confiabilidade na análise do sistema produtivo com foco ambiental. No Brasil, muitas commodities agrícolas já foram avaliadas pelo ACV (DE SOUZA DIAS et al., 2015; KAMALI et al., 2017; REGA; FERRANTI, 2018).

A cultura do tabaco demanda o uso intenso de defensivos agrícolas, de grandes quantidades de fertilizantes, assim como grandes quantidades de lenha para a cura das folhas (HUSSAIN et al., 2017). Ainda, sendo a cultura do tabaco uma atividade agrícola praticada de forma intensa, com grande consumo de insumos e forte ação sobre os ecossistemas em que está inserido, é preciso obter informações confiáveis sobre os impactos causados por esta cultura, tanto sobre o meio ambiente quanto sobre a saúde humana. A Análise de Ciclo de Vida (ACV) é uma ferramenta que utiliza as premissas da sustentabilidade dos processos, auxiliando de forma clara e com grande potencial nas tomadas de decisões relativas aos processos analisados (SAADE et al., 2014), uma vez que esta ferramenta permite uma visão crítica dos processos e seus potenciais impactos ambientais.

A necessidade de suprir uma demanda oriunda da commodity agrícola a qual é uma das bases econômicas da região sul do Brasil apresenta o desafio de realizar um mapeamento dos impactos ambientais da fase de plantio do Tabaco. Para tanto, o presente trabalho tem por objetivo realizar um estudo comparativo dos impactos ambientais produzidos pelas diferentes formas da produção agrícola do tabaco cultivado para comercialização, considerando as diretrizes de plantio requeridas para categoria orgânica e convencional por meio da ferramenta da Análise do Ciclo de Vida. 


\section{METODOLOGIA}

A metodologia utilizada para a realização do presente trabalho foi dividida em duas fases, como podemos visualizar na Figura 1. Na primeira fase ocorreu a caracterização de cada etapa do processo agrícola da produção do tabaco, partindo dos canteiros de produção de mudas até o transporte para comercialização nas empresas fumageiras.

Figura 1 - Fluxograma das fases realizadas no estudo

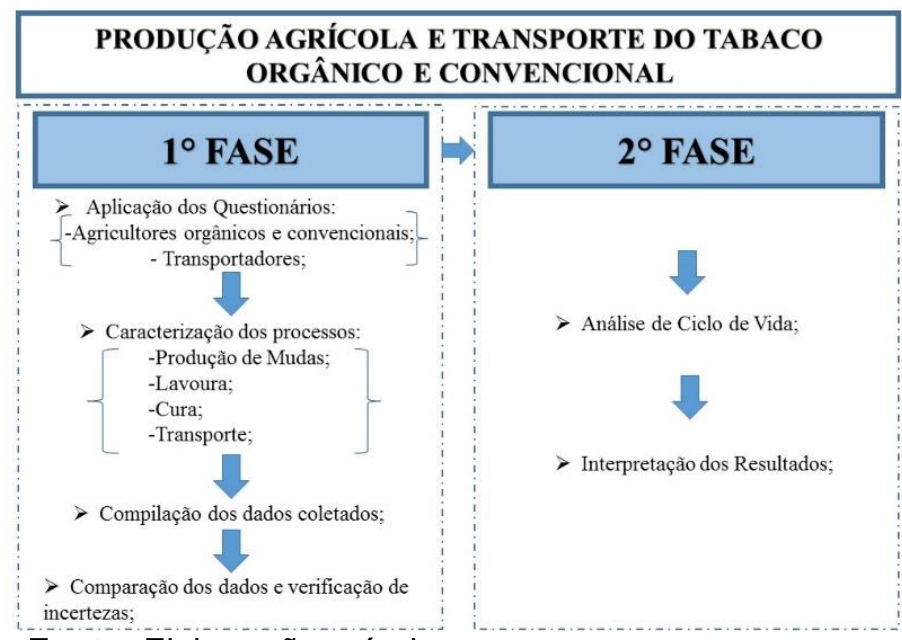

Fonte: Elaboração própria.

A fase seguinte, consta da compilação dos dados coletados na primeira etapa e a sua utilização no software para a análise dos impactos ambientais da produção agrícola do tabaco convencional e orgânico por meio da ferramenta do ACV, para a realização do inventário de emissões para o ambiente e o cálculo dos impactos ambientais associados a cada etapa dos sistemas produtivos, utilizando o método $\mathrm{ReCiPe}$ 2016 Midpoint $(\mathrm{H})$.

A coleta de dados e informações necessárias para este estudo foi realizada pela aplicação direta de dois questionários em produtores do Vale do Rio Pardo- RS, um primeiro direcionado aos agricultores orgânicos e segundo aos convencionais, no qual responderam questões relacionadas a entrada de insumos para a caracterização dos processos, dividida em 3 etapas: produção de mudas, lavoura e cura. Um outro questionário foi direcionado aos transportadores do tabaco, para caracterização desta última etapa avaliada. 
Este banco de dados foi comparado ao fornecido por uma empresa beneficiadora da região, o qual conta com um número de 12.000 produtores pesquisados. Os resultados desta comparação foram satisfatórios uma vez que não houve diferença nos resultados, comprovando a integridade e confiabilidade dos dados coletados. $O$ espaço de tempo na coleta de dados deste estudo atinge todo o ciclo das safras 2015/2016, 2016/2017 e 2017/2018.

A avaliação do ciclo de vida foi realizada seguindo a metodologia padronizada pelas normas ABNT NBR 14.040 e 14.044, ambas de 2009 (ABNT, 2009). O software de simulação foi o SimaPro Release 8.5.0.0, Ph.D Version. O método para seleção das categorias de impacto utilizados foi ReCiPe 2016, que permite a análise de impacto para o nível médio (midpoint), usando a versão $(H)$ hierárquica (hierarchist), para incluir as perspectivas de impacto de longo prazo. O modelo hierárquico foi utilizado por ser o modelo de consenso, normalmente encontrado em modelos científicos e considerado como modelo padrão (WEIDEMA et al., 2013).

O objetivo do método ReCiPe é a transformação da longa lista de resultados do inventario do ciclo de vida (ICV) em um limitado número de indicadores ambientais. O ReCiPe midpoint utiliza um total de 18 indicadores, porém este estudo considerou no total cinco categorias de impacto, detalhadas na Tabela 01.

Tabela 1 - Categorias de impactos e método de avaliação utilizados

\begin{tabular}{lccc}
\hline \multicolumn{1}{c}{ categoria de impacto } & abreviatura & unidade & método \\
\hline Aquecimento Global & PAG & $\mathrm{kg} \mathrm{CO}-\mathrm{Eq}$ & ReCipe midpoint \\
Acidificação Terrestre & $\mathrm{PA}$ & $\mathrm{kg} \mathrm{SO}-\mathrm{Eq}$ & ReCipe midpoint \\
Eutrofização da água doce & $\mathrm{PE}$ & $\mathrm{kg} \mathrm{P}-\mathrm{Eq}$ & ReCipe midpoint \\
Uso da terra & Landuse & $\mathrm{m} 2 \mathrm{a} \mathrm{crop}-\mathrm{Eq}$ & ReCipe midpoint \\
Escassez de recursos fósseis & FRS & $\mathrm{kg} \mathrm{oil-Eq}$ & ReCipe midpoint \\
\hline
\end{tabular}

O programa computacional utilizado utiliza o banco de dados Ecoinvent® 3.5, que não contempla informações relativas à produção de tabaco. Portanto, todas as etapas tiveram de ser incluídas individualmente para o cálculo dos impactos ambientais. Além, disso, o banco de dados praticamente não oferece opções de informações relativas às condições brasileiras.

A primeira fase do estudo corresponde à definição do escopo. Neste estudo o escopo foi considerado desde a produção de mudas de tabaco, até seu transporte a 
empresa, levando em consideração as etapas intermediárias de lavoura e cura (cradle-to-gate).

Neste trabalho a unidade funcional utilizada, corresponde a uma tonelada de tabaco seco, transportado para comercialização até a planta beneficiadora.

Na Tabela 2 está apresentado os dados das quantidades levantadas no inventário e utilizadas no software para a produção de uma tonelada de tabaco seco orgânico e convencional.

Tabela 2 - Dados das quantidades de entradas do inventário para produção de 1 ton de tabaco seco orgânico e convencional

\begin{tabular}{lcccc}
\hline & \multicolumn{2}{c}{$\begin{array}{c}\text { 1ton } \\
\text { Convencional }\end{array}$} & \multicolumn{2}{c}{$\begin{array}{c}\text { 1ton } \\
\text { Orgânico }\end{array}$} \\
\hline Entradas & Quantidade & Unidade & Quantidade & Unidade \\
\hline Sementes & 0,03 & $\mathrm{Kg}$ & 0,03 & $\mathrm{Kg}$ \\
Bandeja isopor & 8,82 & $\mathrm{Kg}$ & 8,82 & $\mathrm{Kg}$ \\
Substrato & 67,82 & $\mathrm{Kg}$ & 94,19 & $\mathrm{Kg}$ \\
Água (poço) & 1,51 & $\mathrm{M}{ }^{3}$ & 1,89 & $\mathrm{M}^{3}$ \\
Arcos metálicos & 39,6 & $\mathrm{Kg}$ & 39,6 & $\mathrm{Kg}$ \\
Inseticida agroquímico & 0,19 & $\mathrm{Kg}$ & 0 & - \\
Herbicida agroquímico & 1,76 & $\mathrm{~L}$ & 0 & - \\
Fungicida agroquímico & 0,39 & $\mathrm{Kg}$ & 0 & - \\
Energia elétrica & 393,60 & $\mathrm{Kwh}$ & 479,54 & $\mathrm{Kwh}$ \\
Calcário & 622,17 & $\mathrm{Kg}$ & 2120,7 & $\mathrm{Kg}$ \\
Lenha & 2,7 & $\mathrm{Ton}$ & 2,7 & $\mathrm{~T}$ \\
Diesel & 35 & $\mathrm{~L}$ & 60 & $\mathrm{~L}$ \\
Fertilizante orgânico & 0 & - & 5555 & $\mathrm{Kg}$ \\
Fertilizante hidrossoluvel 18-8-18 & 1,02 & $\mathrm{Kg}$ & 0 & - \\
Fertilizante base NPK 10-16-08 & 323 & $\mathrm{Kg}$ & 0 & - \\
Fertilizante cobertura NPK 15-05-15 & 93,28 & $\mathrm{Kg}$ & 0 & - \\
Fertilizante Yara Liva 19 Ca NPK 14-00-15 & 32,03 & $\mathrm{Kg}$ & 0 & - \\
Fertilizante salitre do Chile NPK 15-00-14 & 141 & $\mathrm{Kg}$ & 0 & - \\
Anti brotante convencional & 1,27 & $\mathrm{~L}$ & 0 & - \\
Anti brontante orgânico & 0 & - & 25,59 & $\mathrm{Kg}$ \\
Inseticida orgânico & 0 & - & 2,41 & $\mathrm{~L}$ \\
Fungicida orgânico & 0 & - & 0,06 & $\mathrm{~L}$ \\
\hline Fonte: Eataco & & & &
\end{tabular}

Fonte: Elaboração própria.

Para realizar a análise de impacto na etapa de produção das mudas foi considerado o uso de fertilizantes sintéticos para o tabaco convencional e fertilizantes orgânicos para o tabaco orgânico, o uso de substrato, agrotóxicos para o tabaco convencional e inseticidas e fungicidas de origem natural para a produção orgânica, assim 
como as sementes utilizadas. Em relação ao fluxo de materiais foi levado em conta o plástico, isopor e o aço como principais materiais de construção das piscinas.

O consumo de energia para o bombeamento da água até a piscina também foi levado em consideração neste trabalho.

$\mathrm{Na}$ etapa de lavoura, os pontos considerados como entrada para a análise no software foram os agrotóxicos no caso do convencional e inseticidas de origem natural para o tabaco orgânico, os fertilizantes sintéticos (convencional) e adubos orgânicos (orgânico) adicionados ao solo, as mudas do tabaco, a calagem realizada no solo e o consumo de combustível para o preparo da terra.

Já na fase de secagem as entradas do inventário foram iguais para as duas formas de produção, considerando a utilização de lenha e a energia elétrica gasta. No transporte foram quantificados a quilometragem percorrida e o consumo de combustível utilizado no transporte até as empresas beneficiadoras na cidade de Santa Cruz do Sul.

As emissões da folha do tabaco durante o processo de secagem/cura não foram consideradas neste trabalho devido à falta de dados disponíveis na literatura e nos bancos de dados disponíveis.

Emissões de gases de efeito estufa no nível do campo, associadas a fertilizantes aplicado nos dois sistemas de produção de tabaco dependem, principalmente, do tipo de adubo orgânico e fertilizantes sintéticos. Neste estudo, as emissões de gases, decorrentes do uso do estrume orgânico (cama de aviário), levaram em conta os cálculos realizados pelo modelo utilizado. Mesma consideração foi realizada em relação às emissões derivadas dos fertilizantes sintéticos e agrotóxicos. A classificação de agrotóxicos foi realizada a partir dos seus respectivos princípios ativos disponíveis no banco de dados do software

Neste estudo o teor médio de umidade da lenha é de $20 \%$, a densidade média de $375 \mathrm{~kg} / \mathrm{m}^{3}$ e poder calorífico de $3.400 \mathrm{Kcal} \mathrm{kg-1.} \mathrm{Para} \mathrm{a} \mathrm{simulação} \mathrm{da} \mathrm{cura} \mathrm{foi} \mathrm{con-}$ siderado um consumo de $7,2 \mathrm{~m}^{3}$, ou 2,7 ton. de lenha para uma tonelada de tabaco seco, o qual equivale a 39.131 MJ de energia.

A cultura do tabaco ocupa a terra por apenas metade do ano agrícola, fornecendo as terras cultivadas livres para outras culturas no semestre restante. Assim, estas podem se beneficiar de fertilizantes aplicados à cultura do tabaco; no entanto, 
para este estudo, assumimos que todos os impactos ambientais da aplicação de fertilizantes estão associados à produção de tabaco.

\section{DESENVOLVIMENTO}

\subsection{Potencial de aquecimento global}

A Figura 2 apresenta a contribuição das quatro fases dos dois processos produtivos, em relação à categoria de impacto PAG, expressa em kg CO2 eq por tonelada de tabaco seco. O PAG do plantio orgânico e convencional foi de 1876,8 e 1608,1 kg CO2-eq respectivamente. As etapas de produção de mudas e de cura apresentaram valores semelhantes, uma vez que todas as operações envolvidas são iguais para as duas formas de produção. O PAG da etapa de transporte da produção orgânica foi maior que o da produção convencional, uma vez que a produção orgânica é realizada em locais mais afastados, que exigem uma distância média de transporte maior.

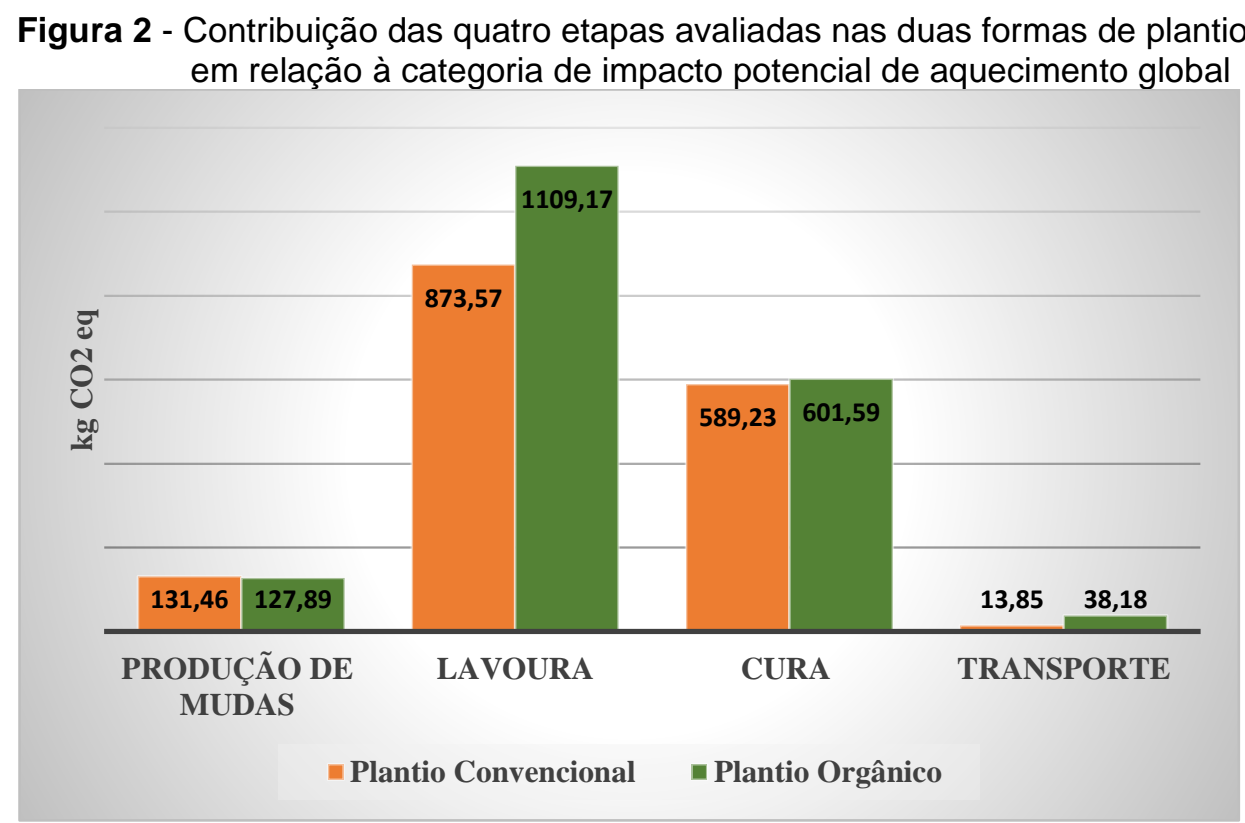

Fonte: Elaboração própria.

$\mathrm{Na}$ Figura 7 se observa que as etapas de lavoura dos dois sistemas foram as que apresentaram a maior contribuição para o PAG, seguidas da etapa de cura, produção de mudas e transporte. As contribuições das etapas de produção de mudas, lavoura, cura e transporte para a produção orgânica foram de 127,89 (6\%), 1109,17 (60\%), 601,59 (32\%) e 38,18 (2\%) kg CO2-eq respectivamente. A contribuição destas 
etapas no plantio convencional foi de 131,46 (8\%), 873,57 (54\%), 589,23 (37\%) e 13,85 (1\%) kg CO2-eq respectivamente.

A análise dos principais processos contribuintes do PAG da etapa de lavoura da produção orgânica demonstrou que este impacto tem grande influência da etapa produtiva do adubo orgânico, principalmente devido à contribuição das emissões relativas à produção da ração para a criação das aves. Outro fator importante são as emissões de NOX, N2O e CO2 relacionadas à aplicação do fertilizante orgânico nas lavouras. Segundo estes autores a emissão de CO2 no solo é a soma da respiração autotrófica, derivada do sistema radicular das plantas e da respiração heterotrófica, derivada de organismos do solo, como micróbios e fauna do solo. Assim, a diferença da emissão de CO2 do solo entre os resíduos orgânicos é decorrente das complexas interações existentes entre os micróbios do solo, a fauna do solo e o sistema radicular após a aplicação de resíduos orgânicos ao solo. Estes autores enfatizam que não somente a emissão de CO2 é estimulada pelos adubos orgânicos. Segundo Li et al. (2018) a adição de resíduos orgânicos igualmente promove a emissão de N2O do solo. Tanto a nitrificação como a desnitrificação são fontes de N2O e podem ocorrer simultaneamente. O processo anterior é dominado por condições aeróbias, enquanto a última é causada principalmente por microrganismos anaeróbios. Devido à entrada de resíduos orgânicos, o aumento da quantidade de componentes orgânicos prontamente degradáveis realça o potencial do processo de nitrificação e da desnitrificação. Dessa forma, a adição de fertilizantes orgânico nas lavouras poderia causar uma maior emissão de GEE do que as lavouras convencionais.

Porém, (AGUILERA et al., 2015) salientam que os resultados dos ACV de sistemas agrícolas devem ser tomados com muito cuidado, uma vez que ainda há muita falta de informação sobre alguns sistemas específicos. Segundo estes autores a maioria dos estudos de avaliação do ciclo de vida de sistema agrícolas empregam os fatores de emissão de N2O de nível 1 do IPCC e/ou não respondem pelo sequestro de $\mathrm{C}$, apesar de que os processos do solo representarem uma grande parcela da pegada de carbono dos sistemas agrícolas e estarem associados a muito alta incerteza (IPCC 2006). Tudo isso pode resultar em estimativas imprecisas do potencial de aquecimento global total. 
A etapa de lavoura no plantio convencional teve como resultado uma emissão de 873,57 kg CO2 equivalente. Este valor de emissão é atribuído à utilização e produção dos fertilizantes nitrogenados e fosfatados que contribuem com o potencial de aquecimento global através da liberação de N2O, CO2 e metano.

$\mathrm{Na}$ etapa de produção de mudas, a fabricação de bandejas de isopor e seu descarte após o uso são um dos maiores contribuintes para o PAG tanto no plantio orgânico como no convencional com emissões principalmente de metano e CO2.

A etapa de cura apresentou resultados significativos para o PAG tanto no plantio de tabaco orgânico $(601,59$ kg CO2 eq), quanto do convencional (589,23 kg CO2 eq). Nesta etapa, a queima de biomassa nas fornalhas das estufas dos produtores rurais para realizar a cura do tabaco, são as causas de maiores liberações de $\mathrm{CO} 2$, $\mathrm{N} 2 \mathrm{O}, \mathrm{CO}$ e $\mathrm{CH} 4$ para a atmosfera. Estes compostos apresentam elevado potencial de aquecimento global, contribuindo significativamente para a categoria avaliada.

O dióxido de carbono proveniente da queima de biomassa é considerado neutro em relação às emissões de GEE, porque o $\mathrm{CO} 2$ produzido pela queima de biomassa é reabsorvido e retido pelas árvores na fase de crescimento, considerando que a biomassa colhida é proveniente de fontes sustentáveis (reflorestamentos para este fim) (ALAM; STARR, 2009; SHARMA et al., 2011). Mas além do CO2, a queima incompleta emite metano, óxidos nitrosos e outros gases com maior potencial de forçamento radiativo do que o $\mathrm{CO} 2$.

$\mathrm{Na}$ etapa de transporte, temos um valor maior para o plantio de tabaco orgânico $51,73 \mathrm{~kg}$ CO2 equivalente, este valor está associado à maior utilização de combustível para o transporte do tabaco orgânico até as empresas beneficiadoras, devido à maior distância entre os produtores de tabaco orgânico e o destino final do transporte de tabaco seco. Geralmente, os processos de transporte com máquinas agrícolas e caminhões geram altas emissões, levando a valores mais altos de PAG. Como pode ser visto na Figura 7, as emissões líquidas no estágio de transporte são inferiores a 1\% do total de GEE para ambas as variedades de tabaco. (HUSSAIN et al., 2017) descobriram que o transporte de tabaco era responsável pela emissão de $80 \mathrm{~kg}$ de CO2 eq por kg de tabaco seco. 


\subsection{Acidificação Terrestre}

A Figura 3 apresenta a contribuição das quatro fases dos dois processos produtivos, em relação à categoria de impacto Potencial de acidificação terrestre, expressa em kg SO2 eq. O PA total do plantio orgânico e convencional foi de 18,1 e 7,7 $\mathrm{kg} \mathrm{SO} 2$ eq respectivamente.

Figura 3 - Contribuição das quatro etapas avaliadas nas duas formas de plantio em relação à categoria de impacto acidificação terrestre

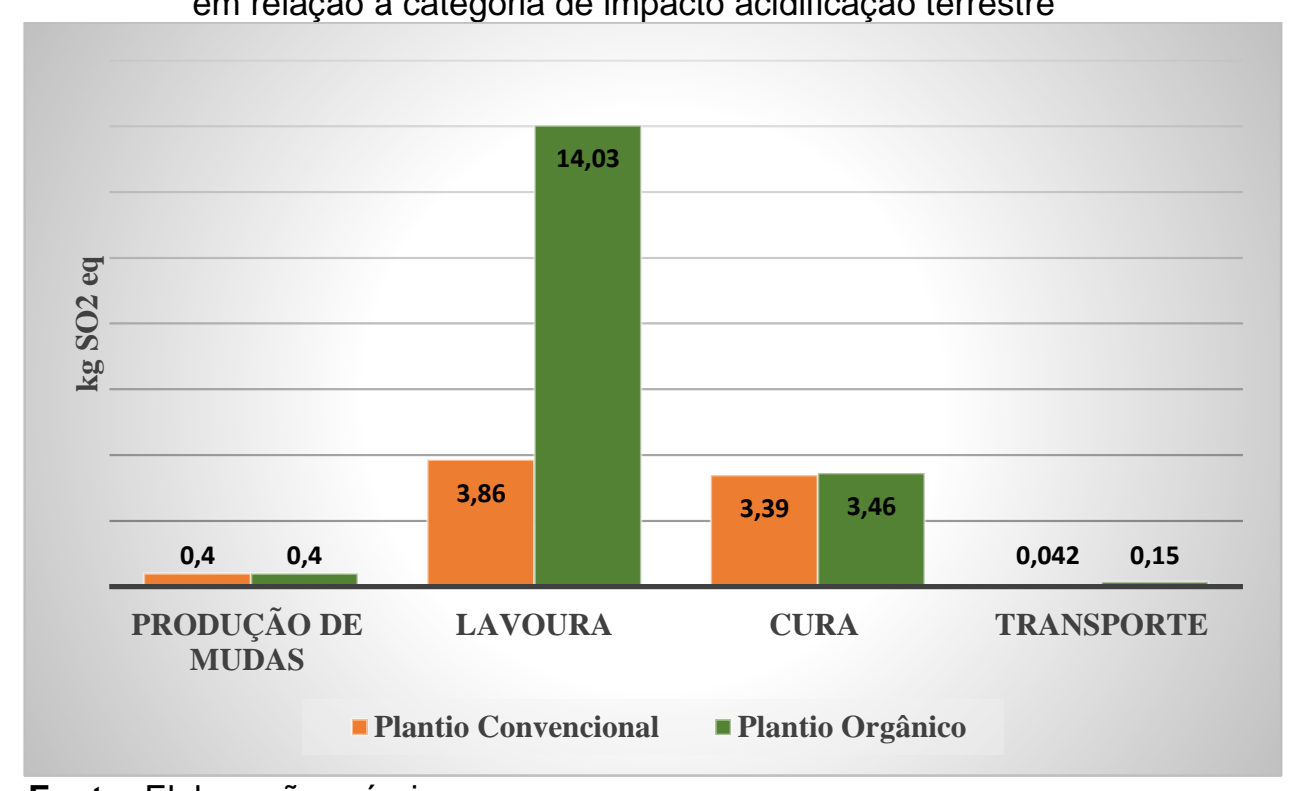

Fonte: Elaboração própria.

A Figura 8 mostra que as etapas de lavoura nos dois sistemas foram as que apresentaram a maior contribuição para o PA, seguidas da etapa de cura, produção de mudas e transporte. As contribuições das etapas de produção de mudas, lavoura, cura e transporte para a produção orgânica foram de 0,4 (3\%), 14,03 (77\%), 3,46 $(19 \%)$ e $0,15(1 \%)$ kg SO2 eq respectivamente. A contribuição destas etapas no plantio convencional foi de 0,4 (5\%), 3,86 (50\%), 3,39 (44\%) e 0,042 (1\%) kg SO2 eq respectivamente.

A etapa que se sobressai é a de lavoura do plantio orgânico, com um valor de 14,03 kg SO2 equivalente. Este valor está associado à produção e utilização dos fertilizantes orgânicos nesta etapa, devido principalmente às emissões de amônia, NOx e SO2. O processo de produção dos fertilizantes sintéticos que é utilizado na etapa de lavoura convencional é a fonte dominante de emissões de $\mathrm{NH} 3$, NOx e SO2, em 
quanto que o seu uso nas lavouras contribui apenas com uma pequena parte deste impacto liberando principalmente amônia, SO2 e N2O para o solo.

De acordo com os dados levantados neste trabalho, são estimados de 7,4 $\mathrm{m}^{3}$ lenha, o equivalente a 2,7 toneladas de biomassa florestal, para a secagem de 1 tonelada de tabaco orgânico e convencional. Analisando o valor das emissões associadas ao valor do potencial de acidificação terrestre da cura do tabaco orgânico 3,46 $\mathrm{kg} \mathrm{SO} 2$ equivalente e tabaco convencional 3,39 kg SO2 equivalente, estes valores estão relacionados às emissões de NO, dióxido de enxofre e amônia, devido à queima de biomassa nas fornalhas das estufas, contribuindo diretamente para o impacto ambiental da acidificação terrestre.

\subsection{Eutrofização da água doce}

A Figura 4 apresenta os valores deste impacto para cada etapa do processo considerado, os valores totais para PE no sistema de plantio orgânico e convencional foram de 0,45 kg P- eq e 0,98 kg P-eq respectivamente. As etapas de lavoura nos dois sistemas foram as que apresentaram a maior contribuição para o $P E$, seguidas da etapa de cura, produção de mudas e transporte. As contribuições das etapas de produção de mudas, lavoura, cura e transporte para a produção orgânica foram de 0,019 (4\%), 0,3 (66\%), 0,13 (29\%) e 0,003 (1\%) kg P-eq respectivamente. A contribuição destas etapas no plantio convencional foi de 0,082 (6,9\%), 0,8 (81\%), $0,12(12 \%)$ e $0,001(0,10 \%) \mathrm{kg} P$ eq respectivamente.

Figura 4 - Contribuição das quatro etapas avaliadas nas duas formas de plantio em Relação à categoria de impacto eutrofização da água doce

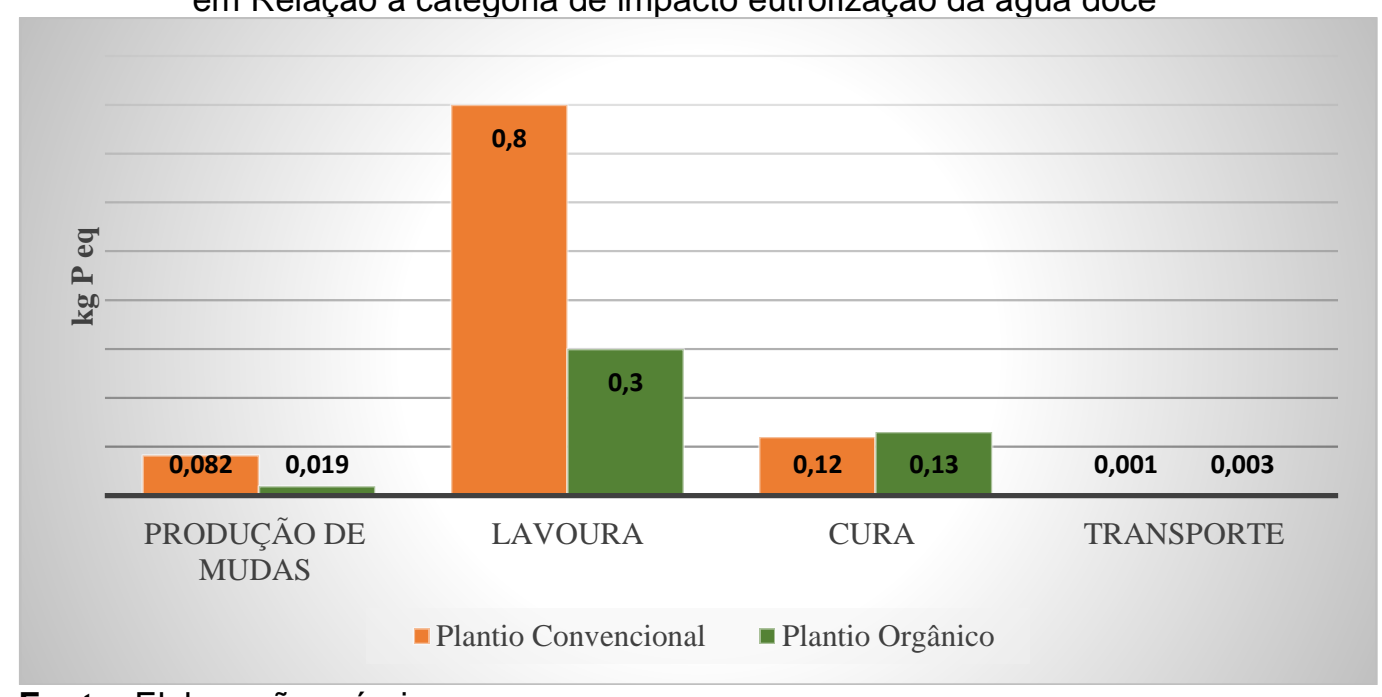

Fonte: Elaboração própria. 
Através da figura e dos valores apresentados é possível verificar que a etapa de lavoura é a que concentra maior potencial de impacto para PE, porém o valor de impacto para o tabaco convencional é notavelmente mais significativo $0,8 \mathrm{~kg} P$ eq, este valor está associado às emissões de fosfato ligado à produção e aplicação dos fertilizantes sintéticos nas lavouras de tabaco convencional.

A individualização das emissões mostra, que o fosfato residual é o principal contribuinte desta etapa. Esse dado evidência que a taxa de aplicação (dosagem) dos fertilizantes fosfatados sintéticos é diretamente proporcional à mudança no potencial de eutrofização. Embora as lavouras de tabaco sejam usadas apenas por meio ano, a outra metade é utilizada por outra lavoura, a qual pode absorver parte destes resíduos de fertilizantes no solo, o que pode diminuir o potencial de eutrofização desta cultura.

\subsection{Uso da terra}

A Figura 5 apresenta a contribuição das quatro fases dos dois processos produtivos, em relação à categoria de impacto Uso da Terra, expressa em kg m2a crop eq. O valor total para o impacto Land use do plantio orgânico e convencional foi de 8625,74 e 6138,14 m2a crop eq respectivamente.

Figura 5- Contribuição das quatro etapas avaliadas nas duas formas de plantio em relação à categoria de impacto uso da terra

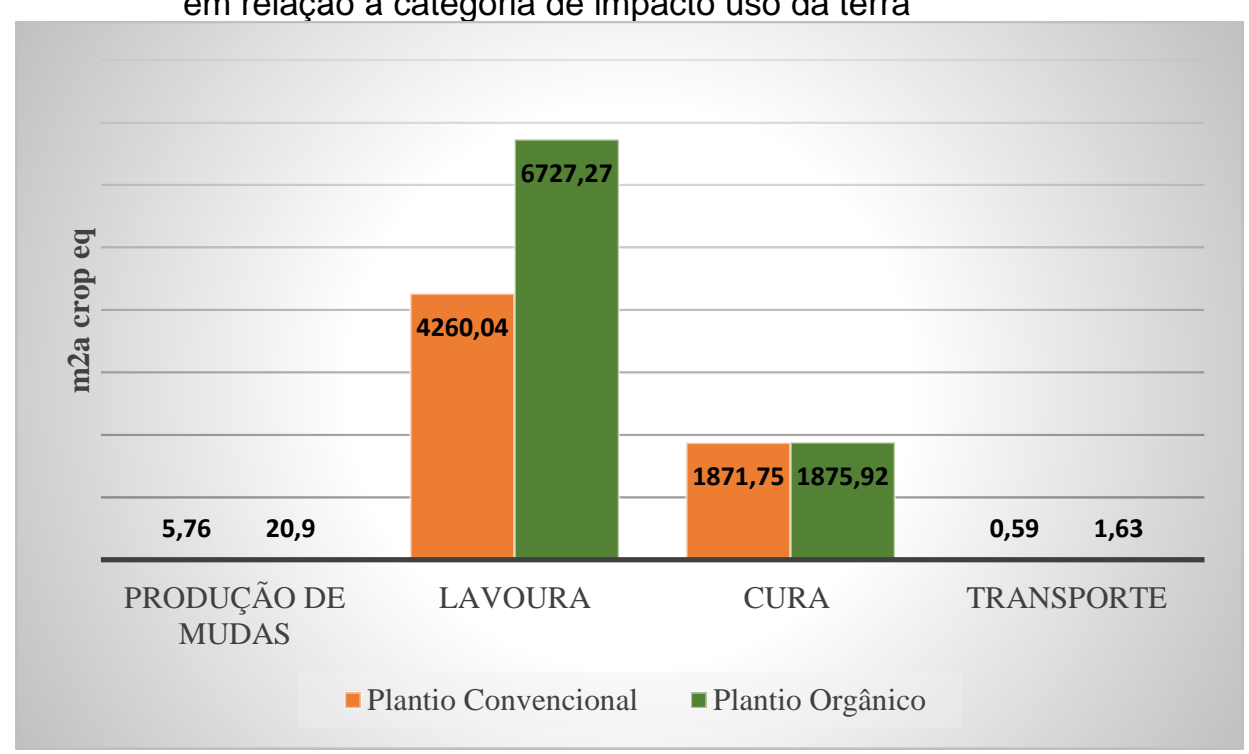

Fonte: Elaboração própria. 
Na Figura 5 é possível observar que as etapas de lavoura nos dois sistemas foram as que apresentaram a maior contribuição para o Land use, seguidas da etapa de cura, produção de mudas e transporte. As contribuições das etapas de produção de mudas, lavoura, cura e transporte para a produção orgânica foram de 20,9 $(0,25 \%)$, 6727,27 (78\%), 1875,92 (21,75\%) e 1,63 (0,01\%) m2a crop eq respectivamente. A contribuição destas etapas no plantio convencional foi de 5,76 (0,00\%), 4260,04 $(70 \%), 1871,75(30 \%)$ e $0,59(0,00 \%)$ m2a crop eq respectivamente.

Os valores da etapa de lavoura nos dois sistemas estão associados à grande utilização dos recursos do solo para os cultivos. Principalmente por que nesta etapa se concentra o revolvimento das terras para o preparo do solo principalmente no sistema de plantio convencional, muitas vezes com a utilização de maquinário agrícola, o que acaba gerando uma mudança na cobertura e na estrutura do solo, acarretando ou tornando mais propenso à lixiviação das partículas e erosão, afetando as espécies terrestres daquele habitat.

O resultado superior para a categoria na etapa de lavoura orgânica é atribuído também em grande parte à maior área de solo necessária para produzir o tabaco orgânico, quando comparado com a produção da mesma quantidade do tabaco convencional. Outro fator que contribuiu para os resultados na etapa de lavoura é a utilização de fertilizantes orgânicos e sintéticos durante a produção do tabaco, toda essa carga de nutrientes acaba afetando e modificando as condições iniciais do solo, além da liberação de compostos, metais pesados e emissões.

\subsection{Escassez de recursos fósseis}

As contribuições das quatro etapas de produção das duas variedades de tabaco sobre o impacto da Escassez de Recursos Fósseis expressas em kg oil eq, são apresentadas na Figura 6. O valor total para o impacto FRS no plantio orgânico e convencional foi de 380,60 e $383,39 \mathrm{~kg}$ oil eq respectivamente.

$\mathrm{Na}$ Figura 6 é possível observar que as etapas de lavoura nos dois sistemas foram as que apresentaram a maior contribuição para a FRS, seguidas da etapa de cura, produção de mudas e transporte. As contribuições das etapas de produção de mudas, lavoura, cura e transporte para a produção orgânica foram de 48,62 (13\%), 
162,08 (42,5\%), 156,65 (41\%) e 13,24 (3,5\%) kg oil eq respectivamente. A contribuição destas etapas no plantio convencional foi de 44,46 (11,5\%), 179,98 (47\%), 154,15 $(40 \%)$ e $4,79(1,5 \%) \mathrm{kg}$ oil eq respectivamente.

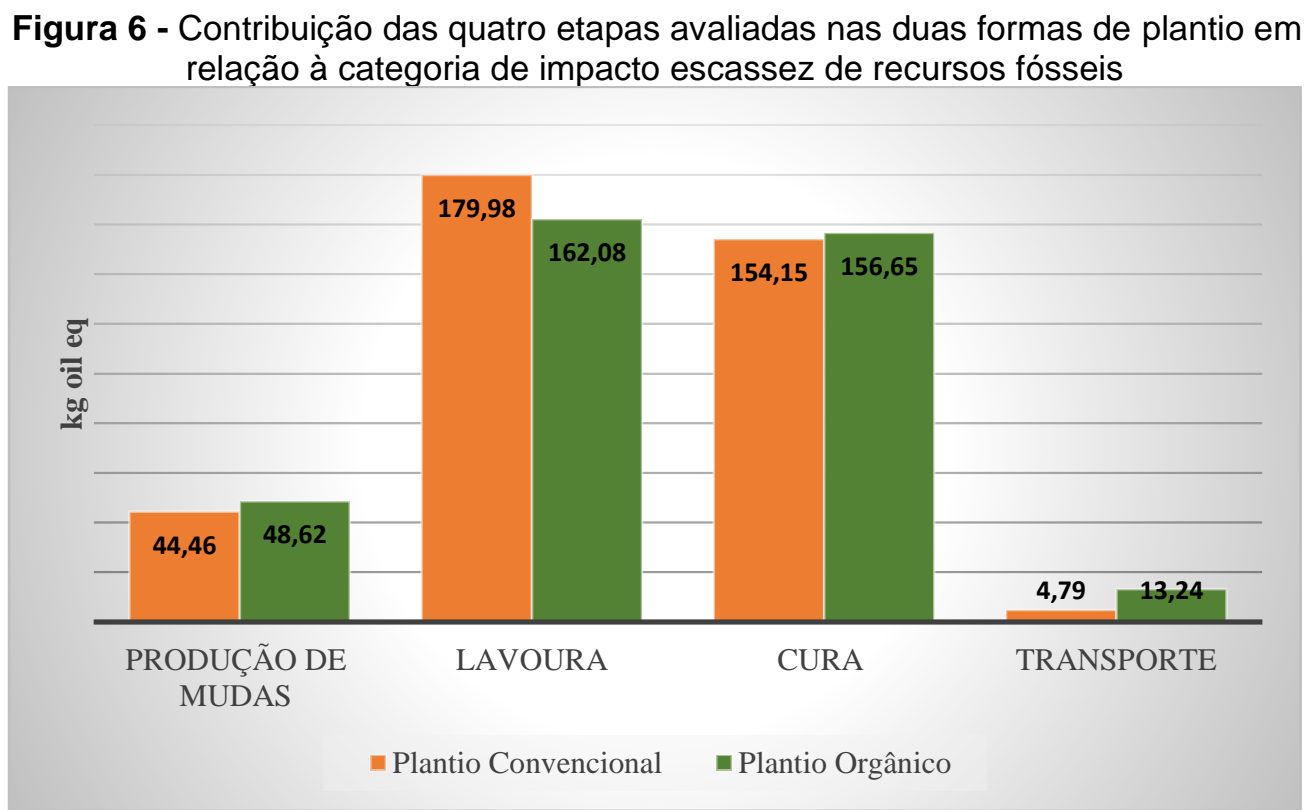

Fonte: Elaboração própria.

A etapa e lavoura convencional é que apresentou os maiores valores $(179,98$ $\mathrm{kg}$ oil eq) este valor está associado, em grande parte, à produção de fertilizantes. $\mathrm{O}$ consumo de combustíveis fósseis na produção de fertilizantes, principalmente o uso de gás natural para a produção de amônia e ácido nítrico, mostra uma influência importante sobre a escassez de recursos fósseis (MAKHLOUF et al., 2015). De acordo com (SKOWROŃSKA; FILIPEK, 2014) a produção de fertilizantes atualmente compreende cerca de $2-3 \%$ do consumo global de energia. Os fertilizantes nitrogenados são responsáveis pela maior parte desse consumo. A maior parte da energia é necessária pela fixação do nitrogênio atmosférico para fabricar amônia. É necessária energia significativa para a conversão de amônia em ureia. Cerca de $77 \%$ da produção mundial de amônia é baseada na reforma a vapor de gás natural, $14 \%$ na gaseificação de carvão e 9\% na oxidação parcial de derivados de petróleo e frações de hidrocarbonetos pesados (BAROSI et al., 2014).

A etapa de cura tem uma contribuição total de 154,15 e 156,65 kg de óleo eq na produção de variedades convencional e orgânico, respectivamente. As emissões 
associadas à esta etapa estão relacionadas à energia consumida no transporte da lenha, manejo florestal e eletricidade usada para injeção de ar quente nos fornos.

A etapa de lavoura orgânica apresentou um valor significativo para a categoria de impacto, este valor foi atribuído à utilização de recursos fósseis principalmente gás natural e carvão para produção da ração, que alimenta as aves para posterior produção da cama de aviário utilizada como adubo orgânico na produção do tabaco.

\subsection{Ecotoxicidade terrestre}

A ecotoxicidade terrestre apresenta os valores de uma acumulação no solo devido à exposição e toxicidade (efeito) de um produto químico. O potencial de toxicidade (TP), expresso em kg de 1,4-diclorobenzeno-equivalente (1,4DCB-eq), é usado como um fator de caracterização a nível médio (HUIJBREGTS et al., 2017). A Figura 11 apresenta a contribuição das quatro fases dos dois processos produtivos, em relação à categoria de impacto EcoT. A EcoT total do plantio orgânico e convencional foi de 1,76 1,4DCB-eq e 2,14 1,4DCB-eq respectivamente.

Figura 8 - Contribuição das quatro etapas avaliadas nas duas formas de plantio em relação à categoria de impacto ecotoxicidade terrestre

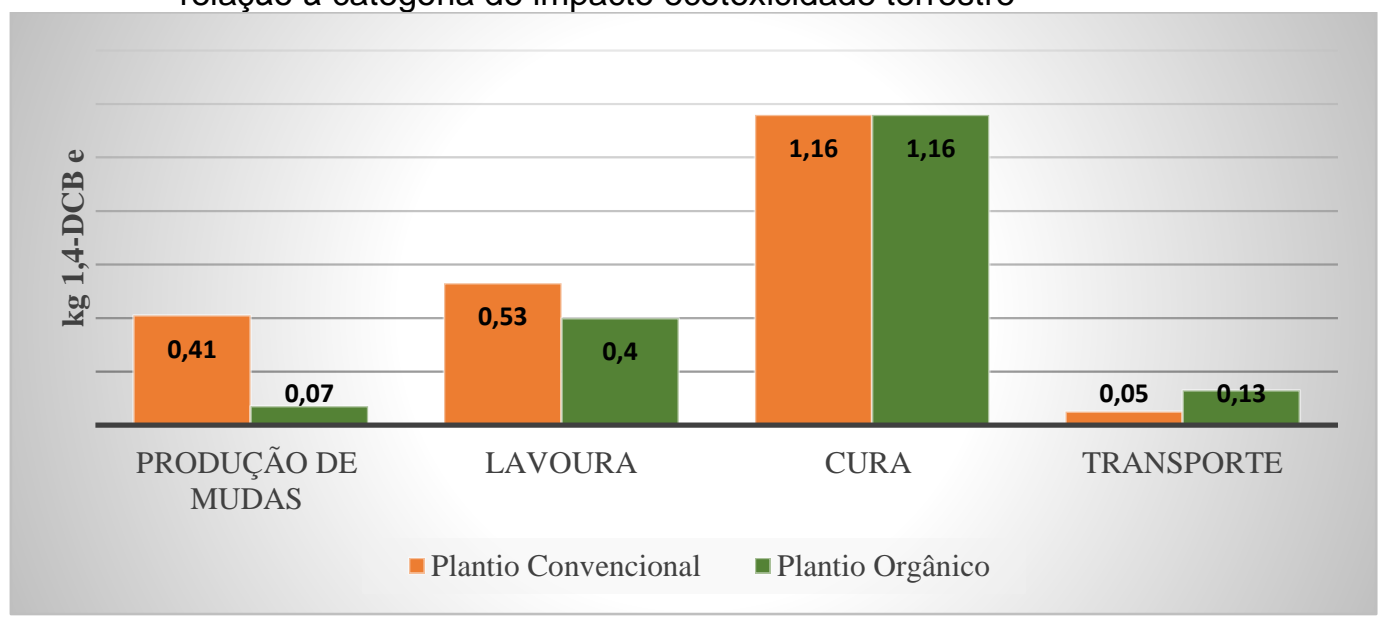

Fonte: Elaboração própria.

Na Figura 11 é possível observar que as etapas de cura nos dois sistemas foram as que apresentaram a maior contribuição para a EcoT, seguidas da etapa de lavoura, produção de mudas e transporte. As contribuições das etapas de produção de mudas, lavoura, cura e transporte para a produção orgânica foram de 0,07 (4\%), 0,4 (23\%), 1,16 (66\%) e 0,13 (7\%) kg SO2 eq respectivamente. A contribuição destas 
etapas no plantio convencional foi de 0,41 (19\%), 0,53 (25\%), 1,16 (54\%) e 0,05 (2\%) kg SO2 eq respectivamente.

Os valores apresentados para a etapa da cura tanto orgânica como convencional do tabaco 1,16 kg 1,4-DCBeq para ambas as formas de cultivo, está relacionado as emissões da queima incompleta de lenha de eucalipto durante a secagem e do uso de um forno de baixa eficiência. Segundo (PIEROBON et al., 2015) a queima de biomassa, embora de origem reflorestada, tem sido associada à emissão de compostos orgânicos com HPA, compostos orgânicos voláteis não-metânicos (COVNMs), emissões orgânicas halogenadas, dioxinas, benzeno e formaldeído, bem como metais pesados que são liberados durante a combustão. Entre os mais perigosos para a saúde humana estão $\mathrm{As}, \mathrm{Ni}, \mathrm{Cr}$, $\mathrm{Cd}$ e $\mathrm{Pb}$. Além daqueles já mencionados, gases como $\mathrm{N}$ (de queima incompleta de combustíveis), $\mathrm{HF}, \mathrm{HCl}$ e amônia também são emitidos durante a combustão.

A etapa de lavoura convencional apresentou um valor de contribuição de 0,53 kg 1,4-DCBeq, predominado pelas emissões dos princípios ativos (dinitroanilina e imidaclopride) dos agroquímicos utilizados nesta etapa.

Os valores de contribuição da etapa de lavoura orgânica foram de 0,4 kg 1,4DCB eq, este foi associado à utilização de fertilizantes orgânicos durantes a etapa, pois a cama de aviário tem uma alta taxa de íons metálicos tendendo a liberar metais pesados e é o único contribuinte para o potencial de eco-toxicidade do solo no sistema orgânico. (MATTSSON; WALLÉN; LONGO et al., 2017) também acreditam que a presença de grandes quantidades de estrume orgânico em sistemas de produção orgânico conduzirá ao aumento gradual do risco de toxicidade do solo, em alguns casos até maior do que sistemas de produção convencionais.

Atualmente, a fim de melhorar a resistência a doenças de gado e aves e para promover seu crescimento rápido, metais pesados como $\mathrm{Cu}$, Zn e As são comumente usados como aditivos em alimentos para a pecuária, como resultado o teor de metal na ração excede a capacidade de absorção digestiva e é excretada pelos animais (WANG et al., 2013).

No estudo realizado por (ZHU et al., 2018), os valores relativos à ecotoxicidade do solo também foram associados aos agroquímicos utilizados no sistema de cultivo convencional da maça, predominando os princípios ativos do carbendazim e etoato, e 
aos fertilizantes orgânicos nos sistema de produção orgânico de maça, devido à emissão de metais pesados, resultados similares com o presente estudo.

\section{CONSIDERAÇÕES}

Com base neste estudo foi possível verificar que as etapas que apresentaram maior impacto nas categorias avaliadas são as de lavoura e secagem, seguidas da etapa de produção de mudas e transporte, nos dois sistemas de plantio avaliados. As principais causas dos impactos, relacionados com os valores calculados são referentes ao uso e produção dos fertilizantes sintéticos e orgânicos, utilização de combustíveis fósseis e recursos fósseis, energia elétrica, emissões da queima da lenha e uso do solo.

Realizando uma comparação geral do plantio orgânico e convencional, é possível verificar que devido à menor produtividade do tabaco orgânico as emissões por unidade de produto acabam se sobressaindo muitas vezes sobre o plantio de tabaco convencional em muitas categorias na maioria das etapas consideradas no estudo.

Os valores expressivos na etapa de lavoura verificados para os impactos de eutrofização de águas doces, acidificação terrestre, potencial de aquecimento global, uso da terra e escassez de recursos fósseis estão relacionados ao uso de fertilizantes sintéticos e orgânicos, agroquímicos e calcário sendo que estes apresentam perdas da massa total aplicada nas lavouras, através das lixiviações, afetando diretamente os ecossistemas mais próximos.

Grande parte dos impactos identificados no estudo estão relacionados à utilização do adubo orgânico nas lavouras, pois este apresenta alto teor de metais, o que acarreta na potencialização de várias categorias de impacto avaliadas, assim, a preocupação em "como" reduzir o impacto ambiental pelos riscos causados pela aplicação de estrume orgânico é uma questão para aumentar os benefícios da produção orgânica de tabaco, diminuindo os impactos associados à adubação.

Em conclusão, este estudo pode ser utilizado como linha de base para estudos de impactos ambientais e confirmou o uso potencial da avaliação do ciclo de vida na determinação da performance ambiental do tabaco convencional e orgânico nas condições do sul do Brasil. Futuras investigações podem otimizar o uso de recursos nos 
subsistemas agrícolas, destacando a necessidade de desenvolver fatores de emissões específicos originados localmente, em condições de clima subtropicais e aprovisionar bancos internacionais com informações regionalizadas.

\section{REFERÊNCIAS}

ASSOCIAÇÃO BRASILEIRA DE NORMAS TÉCNICAS. ABNT 14.044: Gestão ambiental: avaliação do ciclo de vida: requisitos e orientações. Rio de Janeiro: ABNT, 2009a.

ASSOCIAÇÃO BRASILEIRA DE NORMAS TÉCNICAS. ABNT: 14.040. Gestão ambiental: avaliação do ciclo de vida: princípios e estrutura: Rio de Janeiro: ABNT, 2009b.

ASSOCIAÇÃO BRASILEIRA DE NORMAS TÉCNICAS. NBR 46/2011: Gestão ambiental Requisitos gerais dos sistemas orgânicos de produção. Rio de Janeiro: ABNT, 2011.

AGUILERA, E.; GUZMÁN, G.; ALONSO, A. Greenhouse gas emissions from conventional and organic cropping systems in Spain. I. Herbaceous crops. Agronomy for Sustainable Development, v. 35, n. 2, p. 713-724, 2015. https://doi.org/10.1007/s13593-014-0267-9

ALAM, S. A.; STARR, M. Deforestation and greenhouse gas emissions associated with fuelwood consumption of the brick making industry in Sudan. Science of the total environment, v. 407, n. 2, p. 847-852, 2009. https://doi.org/10.1016/j.scitotenv.2008.09.040

BAROSI, R. et al. Mineral nitrogen fertilizers: environmental impact of production and use. Fertil. Compon. Uses Agric. Environ. Impacts, p. 3-44, 2014.

FAO, I. WFP (2014) The state of food insecurity in the world 2014: strengthening the enabling environment for food security and nutrition. FAO, Rome, 2015.

GARNETT, T. Where are the best opportunities for reducing greenhouse gas emissions in the food system (including the food chain)? Food policy, v. 36, p. S23-S32, 2011.

https://doi.org/10.1016/.foodpol.2010.10.010

HUSSAIN, M. et al. Hazardous pollutants emissions and environmental impacts from fuelwood burned and synthetic fertilizers applied by tobacco growers in Pakistan. Environmental Technology \& Innovation, v. 7, p. 169-181, 2017. https://doi.org/10.1016/..eti.2017.02.003

LI, Z. et al. Effects of different agricultural organic wastes on soil GHG emissions: During a 4-year field measurement in the North China Plain. Waste Management, v. 81, p. 202-210, 2018. https://doi.org/10.1016/j.wasman.2018.10.008

MAKHLOUF, A.; SERRADJ, T.; CHENITI, H. Life cycle impact assessment of ammonia production in Algeria: A comparison with previous studies. Environmental Impact Assessment Review, v. 50, p. 35-41, 2015. https://doi.org/10.1016/j.eiar.2014.08.003

MCMICHAEL, A. J. et al. Food, livestock production, energy, climate change, and health. The lancet, v. 370, n. 9594, p. 1253-1263, 2007. https://doi.org/10.1016/S0140$\underline{6736(07) 61256-2}$

SHARMA, B. D.; WANG, J.; LIU, S. Modeling of sustainable biomass utilization and carbon emission reduction. Sensor Letters, v. 9, n. 3, p. 1175-1179, 2011.

https://doi.org/10.1166/sl.2011.1366. 
SKOWROŃSKA, M.; FILIPEK, T. Life cycle assessment of fertilizers: a review. International Agrophysics, v. 28, n. 1, p. 101-110, 2014. https://doi.org/10.2478/intag-2013-0032

TILMAN, D. et al. Forecasting agriculturally driven global environmental change. Science, v. 292, n. 5515, p. 281-284, 2001. https://doi.org/10.1126/science.1057544.

VARGAS, M. A.; OLIVEIRA, B. F. D. Estratégias de diversificação em áreas de cultivo de tabaco no Vale do Rio Pardo: uma análise comparativa. Revista de Economia e Sociologia

Rural, v. 50, n. 1, p. 157-174, 2012. https://doi.org/10.1590/S0103-20032012000100010

WEIDEMA, B. P. et al. Overview and methodology: data quality guideline for the ecoinvent database version 3, 2013.

WIEDMANN, T. O. et al. The material footprint of nations. Proceedings of the National Academy of Sciences, p. 201220362, 2013.

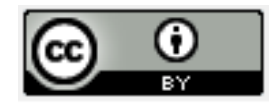

Artigo recebido em: 10/09/2019 e aceito para publicação em: 06/09/2020

DOI: $10.14488 / 1676-1901 . v 20 i 3.3793$ 